\title{
Colorimetric quantification of linking in thermoreversible nanocrystal gel assemblies
}

\author{
Jiho Kang ${ }^{1 \dagger}$ Stephanie A. Valenzuela, ${ }^{2 \dagger}$ Emily Y. Lin ${ }^{1}$, \\ Manuel N. Dominguez, ${ }^{2}$ Zachary M. Sherman, ${ }^{1}$ \\ Thomas M. Truskett, ${ }^{1,3 *}$ Eric V. Anslyn, ${ }^{2 *}$ Delia J. Milliron ${ }^{1,2 *}$ \\ ${ }^{1}$ McKetta Department of Chemical Engineering, University of Texas at Austin, \\ 200 E Dean Keeton St, Austin, Texas 78712, USA \\ ${ }^{2}$ Department of Chemistry, University of Texas at Austin, \\ 2506 Speedway, Austin, Texas 78712, USA \\ ${ }^{3}$ Department of Physics, University of Texas at Austin, \\ 2515 Speedway, Austin, Texas 78712, USA \\ *Corresponding author. Email: milliron@che.utexas.edu (D.J.M); \\ anslyn@austin.utexas.edu (E.V.A); truskett@ che.utexas.edu (T.M.T) \\ $\dagger$ These authors contributed equally to this work.
}

Nanocrystal gel networks can be responsive, tunable materials, but deliberately designing their structure and controlling their properties have been challenging. By employing reversibly bonded molecular linkers, gelation can be realized under conditions predicted by thermodynamics. But, simulations have offered the only microscopic insights, with no experimental means to monitor linking leading to gelation. Here, we introduce a metal coordination linkage with a distinct optical signature allowing us to quantify linking in situ and establish the structural and thermodynamic basis for assembly. Due to coupling between linked indium tin oxide nanocrystals, their infrared absorption shifts abruptly at a chemically tunable gelation temperature. We quantify bonding spectroscopically and use molecular dynamics simulations to under- 


\section{stand bonding motifs as a function of temperature, revealing that gel forma- tion is governed by reaching a critical number of effective links that extend the nanocrystal network. Microscopic insights from our colorimetric linking chemistry enable switchable gels based on equilibrium thermodynamic prin- ciples, opening the door to rational design of programmable nanocrystal net- work assemblies.}

\section{INTRODUCTION}

Colloidal inorganic nanocrystals exhibit distinctive properties, such as localized surface plasmon resonance (LSPR), that can be synthetically tuned by varying size, shape, and elemental composition of their inorganic cores, making nanocrystals attractive building blocks for functional materials (1-3). Nanocrystal assemblies further display collective properties not observed in nanocrystal dispersions, such as LSPR-LSPR coupling, motivating the development of switchable assemblies in which properties can be dynamically modulated based on structure (4-9).

Nanocrystal gels are low density, porous networks with collective properties that depend on structure (10-12). Early approaches to form gels relied on destabilization of nanocrystal colloids by partial stripping of surface-bound ligands (13-16). The resulting gels, formed by nonequilibrium aggregation, displayed assembly-dependent optical and catalytic properties, but offered limited opportunities for structural prediction and control (17). Alternatively, much effort has focused on programmable assembly of nanocrystals functionalized with ligands that terminate in supramolecular recognition groups $(18,19)$. These approaches expand the toolbox of nanocrystal assemblies responsive to chemical and physical stimuli. Still, direct bonding between surface-bound ligands makes it challenging to control structure, often resulting in dense, poorly defined aggregates.

Linking neighboring nanocrystals instead with small molecules offers a convenient, statistical approach to improve structural control in nanocrystal gels $(12,20)$. Past studies explored var- 
ious types of linkers including metal ions $(21,22)$, DNA strands $(23,24)$, and organic molecules forming dynamic covalent bonds $(8,18,25)$. Recently, metal coordination complexes have been used to direct nanocrystal assembly $(18,26-28)$. For example, CdTe nanocrystals capped with tetrazole ligands formed a transparent hydrogel upon the addition of cadmium (II) acetate (27). However, strong chelating agents such as ethylenediaminetetraacetic acid (EDTA) or protonating agents such as trifluoroacetic acid were required to reverse the assembly process $(18,27)$.

Linker-mediated nanocrystal gelation has previously been monitored by changes in structure detected using small-angle X-ray scattering (SAXS) and by the associated modulation of optical properties (e.g., LSPR or excitonic absorption) $(8,21,22,27,29)$. Theoretically predicted phase diagrams can be used to interpret the observed behavior and guide the design of new experiments. Molecular dynamics (MD) simulations of coarse-grained models offer a microscopic view of gelation, including the types and propensities of various linking motifs expected in nanocrystal gels $(8,30-32)$. However, corresponding experimental insights have been lacking, limiting the opportunity to design and understand nanocrystal gels from a molecular perspective. Here, using a linking chemistry with optically quantifiable bonds resolves this limitation and enables us to discern the chemical and thermodynamic basis for gelation, by comparing computational and experimental results across length scales.

In particular, we introduce a thermoreversible and optically responsive gelation strategy using terpyridine-metal complexation to link nanocrystals. Specifically, tin-doped indium oxide (ITO) nanocrystals were functionalized with terpyridine-terminated ligands (TL) and dispersed in $N, N$-dimethylformamide (DMF) with $\mathrm{Co}^{2+}$ and excess $\mathrm{Cl}^{-}$. The formation of cobalt(II)(bis)terpyridine $\left(\mathrm{Co}(\mathrm{Tpy})_{2}\right)$ links between nanocrystals results in gelation at room temperature, causing a red shift in the infrared LSPR absorption due to coupling. Higher temperatures favor dissociation of the linking bonds, releasing $\mathrm{Co}^{2+}$ to form cobalt(II) tetrachloride, reversing the nanocrystal assembly and recovering the higher energy LSPR of dispersed nanocrystals. Importantly, as we show, the concentration of $\mathrm{Cl}^{-}$controls the gelation temperature. By spectral deconvolution, we quantify link formation and observe that gelation is governed by the number 
of links surpassing a threshold, regardless of $\mathrm{Cl}^{-}$concentration. Quantitative analysis of bonding equilibria confirms that a favorable bonding enthalpy controls linking, with $\left[\mathrm{Cl}^{-}\right]$shifting the equilibrium according to Le Chatelier's Principle. MD simulations establish that bonding increases continuously as temperature decreases, but only beyond a critical threshold do large clusters consistent with gelation form. Having ratioanlized the basis for the tunable infrared optical switching, this linking gel strategy may be extended in the future to design nanocrystal assemblies with a wide range of dynamic properties.

\section{RESULTS}

\section{Ligand design and nanocrystal functionalization}

We designed and synthesized terpyridine-terminated ligands (TL) that can facilitate nanocrystal gelation by forming cobalt(II)-(bis)terpyridine complexes (Fig. 1A and figs. S1-S8 and S13). The ligands consist of three domains: a terpyridine moiety on the terminal end used for linking, a PEG-based backbone for nanocrystal dispersibility, and a tricarboxylic acid group for binding to nanocrystal surfaces. ITO nanocrystals were chosen as building blocks because of their infrared LSPR absorption, which is known to shift due to coupling in assemblies $(2,7,8,33)$. The nanocrystals were synthesized via a modified slow growth method (34) and functionalized with TL via direct ligand exchange (8).

Functionalization of ITO nanocrystals with TL had no discernible effect on nanocrystal morphology or plasmonic properties. The size and shape of nanocrystals were unchanged by the exchange of native oleate ligands with TL (Fig. 1B and fig. S14), with the nanocrystal core size being $11.7 \mathrm{~nm} \pm 1.0 \mathrm{~nm}$ (fig. S15). Dispersions of oleate-capped and TL-capped ITO nanocrystals (OA-ITO and TL-ITO, respectively) exhibited nearly identical LSPR absorption (Fig. 1C). The presence of bound TL on nanocrystals was confirmed by comparing ${ }^{1} \mathrm{H}$ NMR spectra of TL and TL-ITO (Fig. 1D and fig. S16). TL displayed a broad peak at 12.5 ppm assigned to the carboxylic acid proton and multiple peaks from 7.4 to $8.8 \mathrm{ppm}$ characteristic of terpyridine $(35,36)$. Meanwhile, TL-ITO showed no sign of the carboxylic acid proton, consis- 


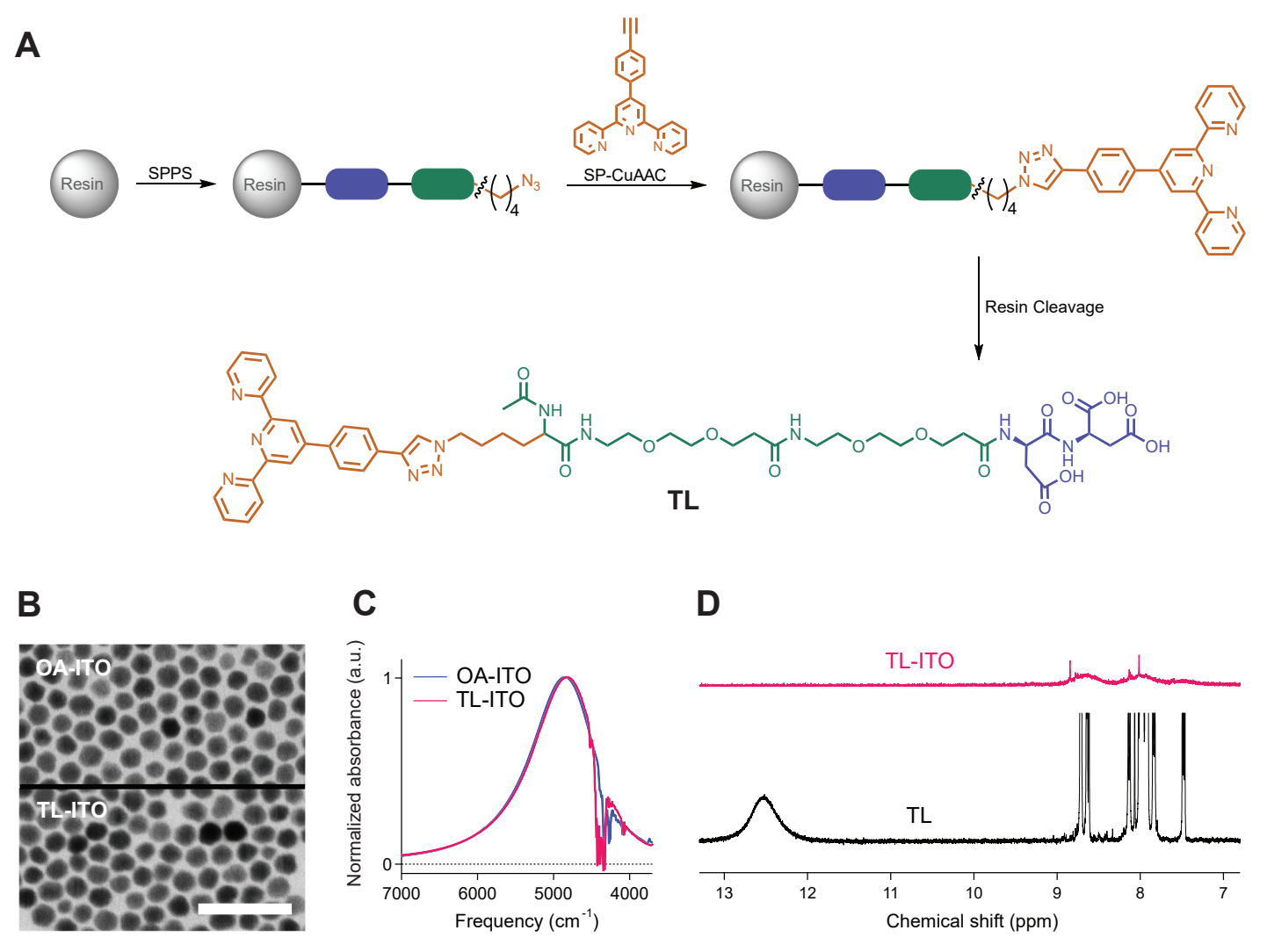

Fig. 1. Synthesis of TL and characterization of TL-ITO. (A) Synthetic scheme of TL showing the terpyridine functional group (orange), PEG backbone (green), and tricarboxylic binding domain (blue). (B) Scanning transmission electron microscopy (STEM) images of nanocrystals capped with native oleate ligands (top) and after TL-functionalization (bottom). Scale bar is $50 \mathrm{~nm}$. (C) LSPR absorption spectra of oleate-capped ITO nanocrystals (blue, in hexane) and TL-ITO dispersion (magenta, in DMF). (D) ${ }^{1} \mathrm{H}$ NMR spectra of TL (black) and TL-ITO (magenta) in deuterated DMSO.

tent with surface-bound carboxylates and no traceable amount of unbound TL was present. The aromatic peaks of terpyridine were significantly broadened and shifted in TL-ITO, consistent with ligands binding to nanocrystals (37). The presence of bound ligands was also confirmed with Fourier transform infrared (FTIR) spectra, where the characteristic vibrational modes of the terpyridine group were visible in the fingerprint region (fig. S17). The $\mathrm{C}=\mathrm{O}$ stretching mode for carboxylic acid groups in TL is absent for TL-ITO, consistent with carboxylate-mediated bonding on the nanocrystal surfaces.

To estimate the average number of TL per nanocrystal, ${ }^{19} \mathrm{~F}$ NMR spectroscopy was carried out on nanocrystals functionalized with a fluorine-tagged $\mathbf{T L}\left(\mathbf{T L}_{\mathbf{F - t a g}}\right)$ in deuterated DMF (figs. 
S9-S11 and S18). Functionalized nanocrystals were dispersed together with an internal reference at known concentration $\left(\mathrm{CFCl}_{3}, 10 \mathrm{mM}\right)$ and the concentration of ligands was calculated by comparing the integrated peak areas, resulting in an average number of $560 \pm 7 \mathbf{T L}_{\mathbf{F} \text {-tag }}$ per nanocrystal.

\section{Thermoreversible nanocrystal assembly}

Adding $\mathrm{CoCl}_{2}$ to a dispersion of TL-ITO rapidly induced the formation of a gel, while a flowing dispersion was recovered upon heating. Previous studies showed that metal-terpyridine complexation can be used to induce nanocrystal aggregation $(18,26,38,39)$. Reversing the assembly process, however, required addition of either a strong chelating agent (EDTA) or strong acid (18). Furthermore, using such chemical stimuli results in the accumulation of byproducts, severely limiting the opportunity to reversibly cycle between assembled and dispersed phases (27). Our key insight to overcome this challenge was to balance the metal-terpyridine bonding equilibrium by adding $\mathrm{Cl}^{-}$(tetrabutylammonium chloride, $\mathrm{TBACl}$ ) to favor the release of $\mathrm{Co}^{2+}$, forming cobalt(II) tetrachloride in a ligand exchange reaction. As a result, thermoreversible ITO nanocrystal gel assemblies were realized based on the dynamic equilibrium between cobalt(II)-(bis)terpyridine complexes and cobalt(II) tetrachloride complexes (bound and free $\mathrm{Co}^{2+}$, respectively) (Fig. 2A).

At relatively low temperature, bound $\mathrm{Co}^{2+}$ is enthalpically favored over free $\mathrm{Co}^{2+}$ and guides nanocrystal assembly by linking together TLs on neighboring nanocrystals. As temperature increases, however, the balance shifts from bound $\mathrm{Co}^{2+}$ to free $\mathrm{Co}^{2+}$, reversing nanocrystal assembly. Theremoreversible nanocrystal assembly was observable by visual inspection (Fig. 2B and fig. S19). A dispersion of TL-ITO immediately formed a gel upon adding $\mathrm{CoCl}_{2}$ at room temperature. The characteristic visible absorption of bound $\mathrm{Co}^{2+}$ made the nanocrystal gel look orange $(40,41)$. The gel repeatedly turned into a flowing dispersion upon heating $\left(90{ }^{\circ} \mathrm{C}\right)$ and back into a gel upon cooling to room temperature. STEM imaging of a dried gel showed an open porous network formed by interconnected nanocrystal clusters (Fig. 2C and fig. S20). Almost 

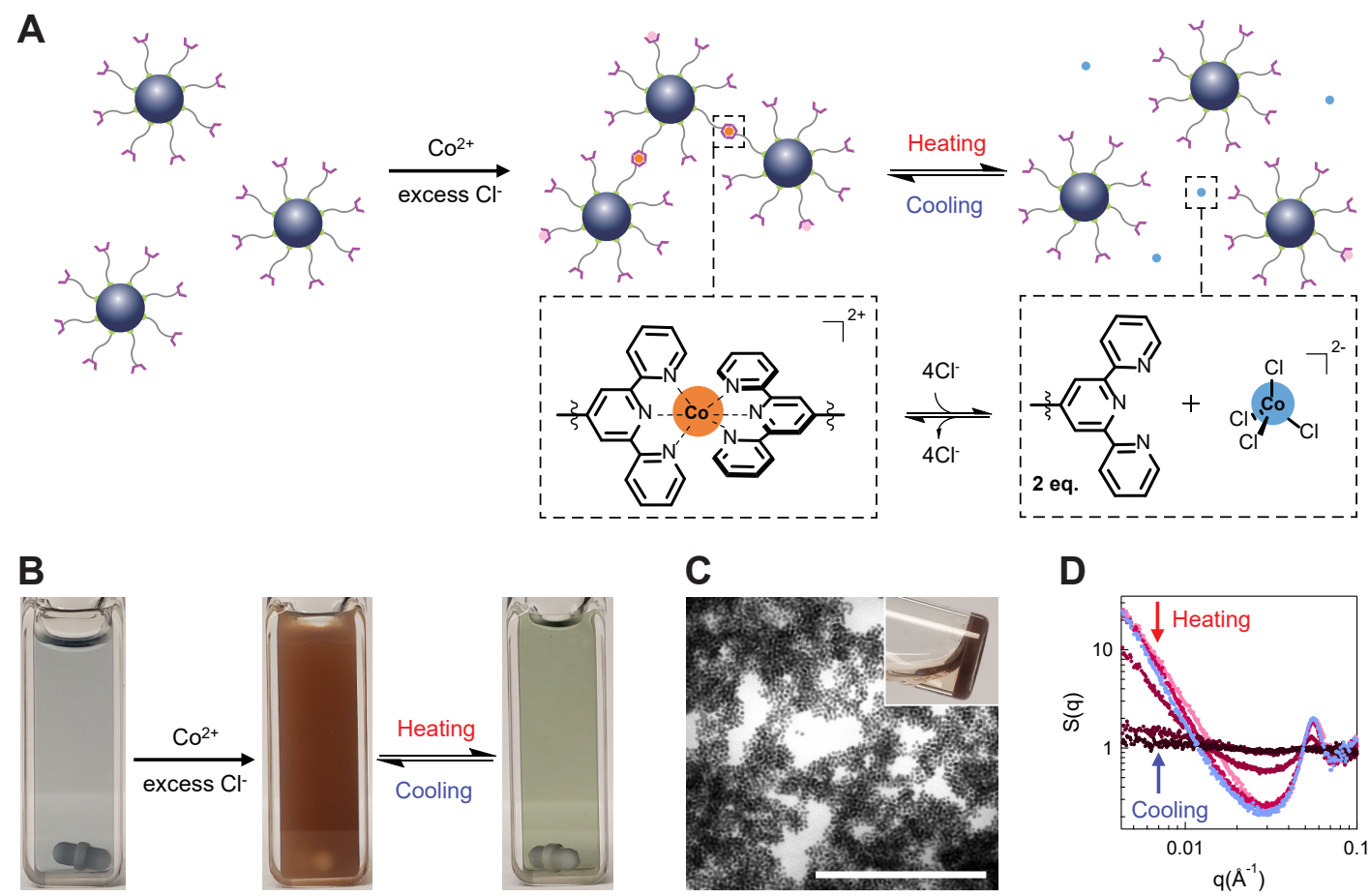

Fig. 2. Thermoreversible nanocrystal assembly. (A) Schematic of thermoreversible nanocrystal assembly controlled by the equilibirum between cobalt(II)-(bis)terpyridine and cobalt(II) tetrachloride complexes. (B) Photo of a TL-ITO dispersion in DMF (left), a nanocrystal gel forming upon the addition of $\mathrm{Co}^{2+}$ (middle) and a free-flowing nanocrystal dispersion at $90{ }^{\circ} \mathrm{C}$ (right). (C) STEM image and photo (inset) of a nanocrystal gel. Scale bar is $500 \mathrm{~nm}$. (D) in situ SAXS structure factor of nanocrystal assembly at different temperatures.

all nanocrystals are incorporated in the gel phase, leaving a clear supernatant (Fig. 2C, inset). Based on the gel volume, the estimated volume fraction of nanocrystals is about $1.2 \%$, so it remains highly porous. To monitor structural changes, in situ SAXS was performed at different temperatures (Fig. 2D). At room temperature, the nanocrystal gels exhibited a characteristic increasing structure factor $S(q)$ at low $q$, indicative of mesoscale organization of nanocrystals with a fractal dimension of 2.2-2.4 (fig. S21). With increasing temperature, $S(q)$ at low $q$ decreased in intensity, approaching unity, consistent with the transition from gel into discretely dispersed nanocrystals. Finally, the characteristic gel $S(q)$ was recovered when cooled to room temperature, indicating thermoreversible gelation.

\section{Tunable and reversible optical modulation}


Gelation brings the ITO nanocrystals close together, producing a substantial shift in infrared absorption due to LSPR-LSPR coupling. LSPR absorption spectra of TL-ITO dispersions containing $\mathrm{Co}^{2+}$ and excess $\mathrm{Cl}^{-}$were monitored in situ at different temperatures by FTIR (Fig. 3 and figs. S22 and S23). At $100{ }^{\circ} \mathrm{C}$, the spectrum closely resembled that of a TL-ITO disper-

A

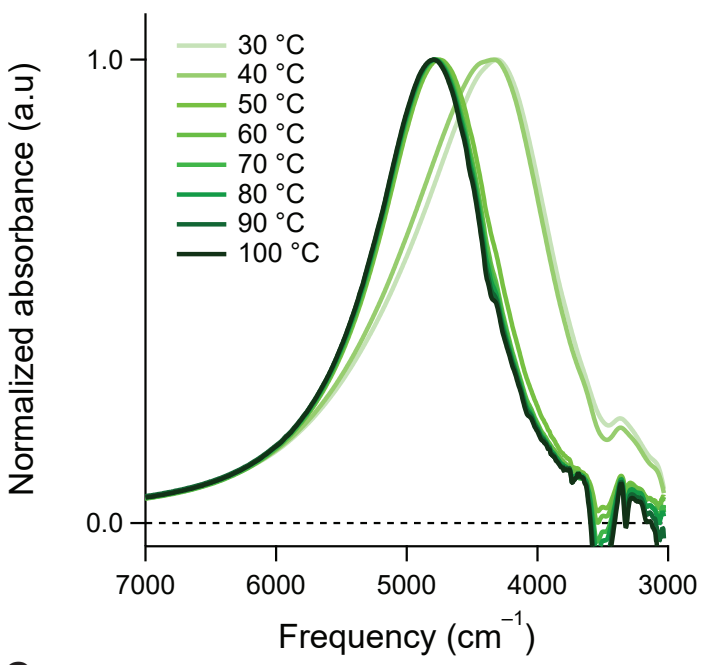

C

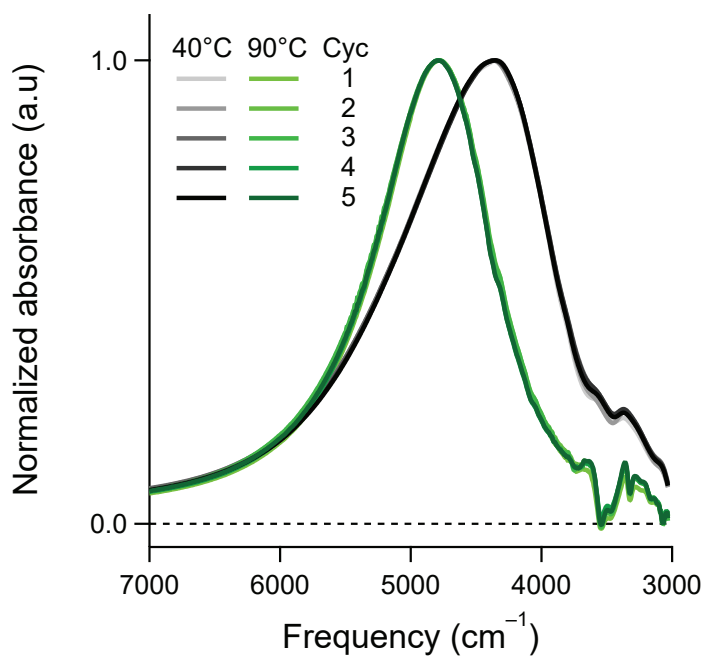

B

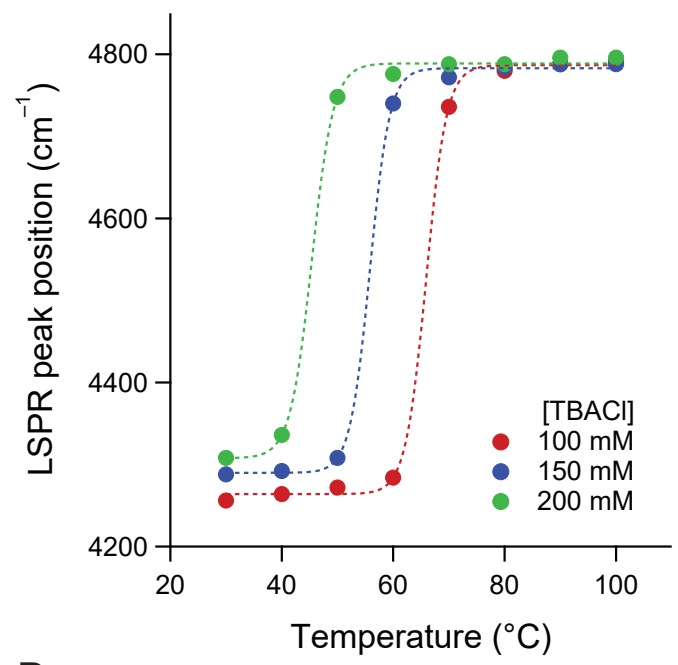

D

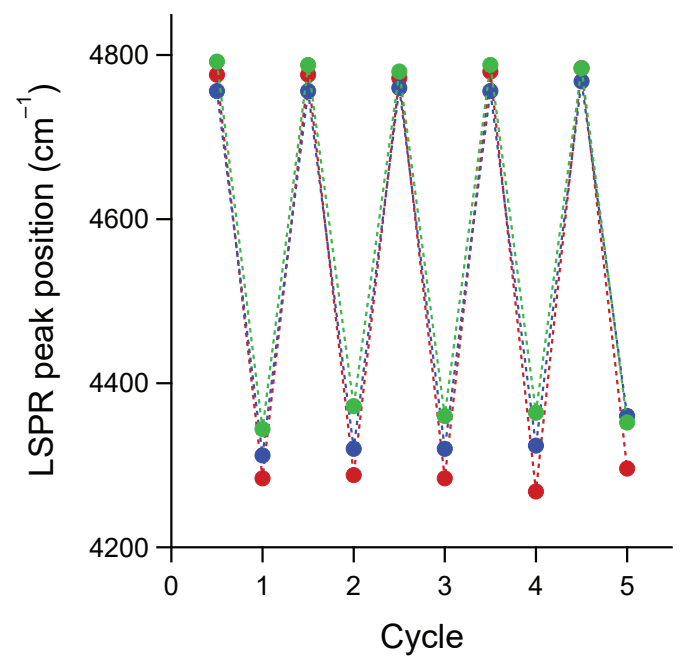

Fig. 3. Infrared optical modulation by thermoreversible nanocrystal assembly. (A) LSPR absorption spectra and (B) LSPR peak position of thermoreversible nanocrystal assembly in DMF at varying temperatures. (C) LSPR absorption spectra and (D) LSPR peak position of thermoreversible assembly in DMF during 5 cycles of heating and cooling. For clarity, only LSPR spectra of the $[\mathrm{TBACl}]=200 \mathrm{mM}$ sample are shown in $(\mathbf{A})$ and $(\mathbf{C})$.

sion without any linker, indicating nanocrystals are well-dispersed. As temperature decreased, however, an abrupt redshift and broadening occured, attributed to coupling between assembled 
nanocrystals $(2,42)$. The phase transition from dispersion to gel was identifiable by the shift of LSPR peak frequency from above $4700 \mathrm{~cm}^{-1}$ to below $4400 \mathrm{~cm}^{-1}$, with the gelation temperature $\left(T_{g e l}\right)$ determined by the inflection point of a sigmoid fit (Fig. 3B). The gelation temperature was tuned by manipulating the equilibrium between $\mathrm{Co}(\mathrm{Tpy})_{2}$ links and free $\mathrm{CoCl}_{4}{ }^{2-}$ ions. Specifically, varying [TBACl] between 100 and $200 \mathrm{mM}$ caused $T_{\text {gel }}$ to shift by more than $20{ }^{\circ} \mathrm{C}$. The reversible nature of the metal coordination links led to highly reproducible gelation upon repeated thermal cycling (Fig. 3, C and D). Through five cycles of heating and cooling, the LSPR spectra were consistent in both dispersion $\left(90^{\circ} \mathrm{C}\right)$ and gel states $\left(40^{\circ} \mathrm{C}\right)$, for all samples.

\section{Quantifying the links during nanocrystal assembly}

Besides changes in LSPR, the visible absorption spectra change markedly during heating, providing a spectroscopic signature of metal coordination linking. Two absorption peaks at 448 and $523 \mathrm{~nm}$ are indicative of the $\mathrm{d}-\mathrm{d}^{*}$ transition of bound $\mathrm{Co}^{2+}$, while the band between 550 and $750 \mathrm{~nm}$ is attributed to the $\mathrm{d}-\mathrm{d}^{*}$ transition of free $\mathrm{Co}^{2+}$. The spectroscopic signals for bound and free $\mathrm{Co}^{2+}$ were consistently reproduced over ten cycles between 90 and $40{ }^{\circ} \mathrm{C}$ (fig. S24). The thermal recyclability of the linking process stands in contrast with the use of chemical stimuli to reverse nanocrystal assembly, which often leads to the accumulation of byproducts and offers limited cyclability.

To quantify bonding, the visible absorption spectra were deconvoluted into three contributions: ITO nanocrystals (bandgap and LSPR), bound $\mathrm{Co}^{2+}$, and free $\mathrm{Co}^{2+}$ (Fig. 4A and figs. S25 and S26). The fraction of free $\mathrm{Co}^{2+}$ was found by comparing the deconvoluted spectroscopic contribution of free $\mathrm{Co}^{2+}$ in the flowing nanocrystal dispersions to that in a control sample lacking terpyridine terminal groups on the ligands (figs. S12 and S13).

Although gelation occurred abruptly at a distinct temperature, the number of links (bound $\mathrm{Co}^{2+}$ ) increased continuously as the temperature was lowered from $100{ }^{\circ} \mathrm{C}$ (Fig. 4B). The quantification of links below the gelation temperature was not possible due to the severe scattering of the gels in the visible region. The fraction of bound $\mathrm{Co}^{2+}$ at the gelation threshold was nearly 
A

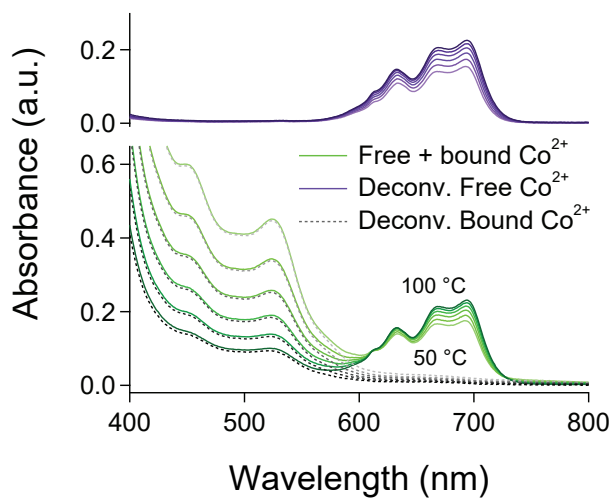

B

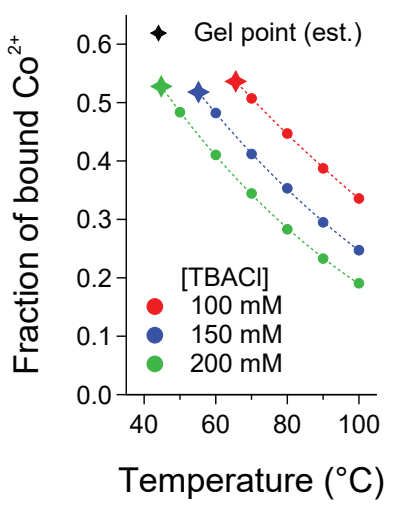

C

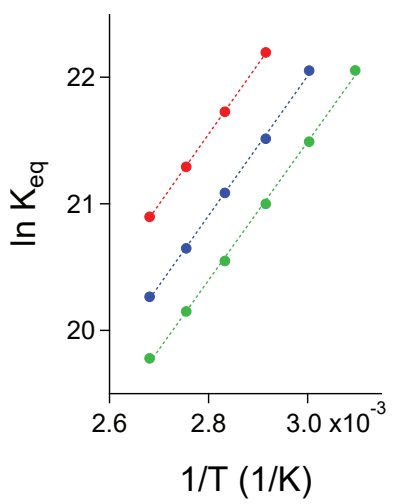

Fig. 4. Quantifying links in thermoreversible nanocrystal assembly. (A) Deconvolution of visible absorption spectra of a flowing nanocrystal dispersion with $[\mathrm{TBACl}]=200 \mathrm{mM}$ : total absorbance of free and bound $\mathrm{Co}^{2+}$ complexes (solid green), deconvoluted contribution from free $\mathrm{Co}^{2+}$ complexes (solid purple), and from bound $\mathrm{Co}^{2+}$ complexes (dashed black). The absorption background due to TL-ITO was subtracted before deconvolution. (B) Fraction of bound $\mathrm{Co}^{2+}$ complexes determined by deconvolution. Gel points are estimated by extrapolating the trendlines to the gelation temperatures obtained from Fig. 3B. (C) van 't Hoff analysis of the equilibrium constant $K_{e q}$ based on concentrations extracted from the spectroscopic deconvolution.

constant at about $53 \%$ (133 bound $\mathrm{Co}^{2+}$ per nanocrystal) regardless of [TBACl], implying that gelation occurs when the average number of links per nanocrystal surpasses a threshold. Our previous study using an aldehyde-hydrazide reversible covalent bonding pair showed that most links formed using bifunctional linkers are "self-links" — in which two ligands on a single nanocrystal are connected by a linker, without effectively extending the nanocrystal network ( 8$)$. Thus, above the gelation temperature, self-links may account for the gradual increase of bound $\mathrm{Co}^{2+}$ with decreasing temperature while the nanocrystals remain dispersed.

To better understand the bonding equilibria and the distribution between self-links and effective links (those that bridge between nanocrystals and extend the network), two types of MD simulations were performed over a range of temperatures above and below $T_{\text {gel }}$ (fig. S27). First, in single nanocrystal simulations, the fraction of bound linker increased continuously with decreasing temperature approaching a fully bound state (Fig. 5A). Subsequently, the fraction of bound linker was computed in 100-nanocrystal systems at four different temperatures, which showed quantitative agreement with the single-nanocrystal calculations, as well as with the cor- 
responding experiments, which collapse to a single curve when temperature is normalized by $T_{\text {gel }}$ (Fig. 5, A and B). This correspondence suggests that the thermodynamics of link formation are largely independent of linking motif, whether self-linking or bridging. In the model, a gelation threshold of $T_{g e l}=0.093 \varepsilon_{b} / k_{\mathrm{B}}$, where $2 \varepsilon_{b}$ is the bonding energy to form a link and $k_{\mathrm{B}}$ is the Boltzmann constant, ensures that the fraction of bound $\mathrm{Co}^{2+}$ matches the experimental value of $53 \%$ at the gelation temperature. Considering an experimental $T_{g e l}$ of $50{ }^{\circ} \mathrm{C}$, this temperature scaling corresponds to a bonding energy to form a link of $57.8 \mathrm{~kJ} \mathrm{~mol}^{-1}$.

Starting from initial configurations of only free linkers, effective links increase over simulation time (Fig. 5C) and the prevalence of effective links, redundant links, and self-links all increased with decreasing temperature (fig. S28). Most of the bound $\mathrm{Co}^{2+}$ participated in selflinks with only a small fraction forming effective links (up to $8 \%$ ), regardless of temperature (Fig. 5C). This result is consistent with our prior observations of linker-mediated nanocrystal gelation, which showed the majority of linkers are "wasted" in self-linking motifs $(8,30,32)$. As temperature dropped to near $T_{\text {gel }}$, the number of effective links crossed a threshold sufficient to induce nanocrystal assembly; slightly more than one effective link per nanocrystal was required to form a percolated network (Fig. 5C), and large, connected clusters of nanocrystals were observed to assemble (Fig. 5, B and D, and video S1 and S2).

Based on the spectroscopic deconvolution, higher concentration of $\mathrm{Cl}^{-}$reduced the fraction of bound $\mathrm{Co}^{2+}$ at each temperature, ultimately lowering $T_{\text {gel }}$. This trend can be explained by the thermodynamic equilibrium between bound and free $\mathrm{Co}^{2+}$, which is driven toward forming more free $\mathrm{Co}^{2+}$ at higher concentration of $\mathrm{Cl}^{-}$. As a result, samples with higher [TBACl] required further cooling to reach the threshold point, leading to lower $T_{g e l}$.

The spectroscopically quantified trends in bound $\mathrm{Co}^{2+}$ with temperature and [TBACl] were used to derive the thermodynamic parameters controlling coordination bonding and thereby directing nanocrystal gelation. Briefly, equilibrium constants $\left(K_{e q}\right)$ at different conditions were obtained using the simplified chemical reaction for linking in our system:

$$
2 \mathrm{TL}+\left[\mathrm{CoCl}_{4}\right]^{2-} \rightleftharpoons\left[\mathrm{CoTL}_{2}\right]^{2+}+4 \mathrm{Cl}^{-}
$$



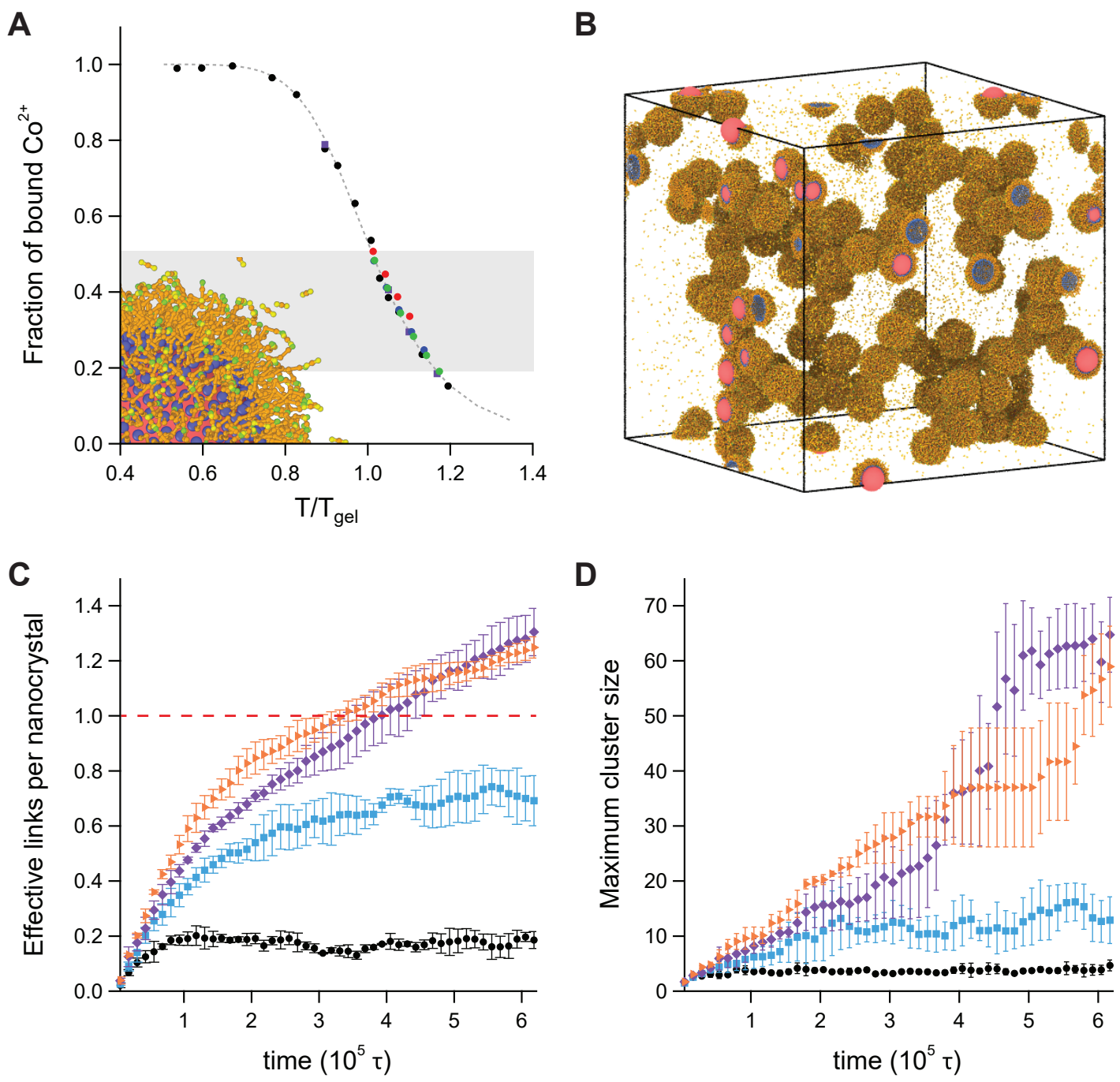

Fig. 5. MD simulations of nanocrystal-linker assembly. (A) Fraction of bound linkers for single nanocrystal (black dots), 100-nanocrystal simulations (purple squares) and [TBACl] = 100 (red dots), 150 (blue dots), and $200 \mathrm{mM}$ (green dots) samples. The shaded region indicates the range of experimental measurements. A snapshot of a single nanocrystal simulation includes representations of the nanocrystal (red), bound ends of the ligands (blue), ligand backbones (orange), and bonding sites (yellow and green). (B) Snapshot of the simulation of a 100-nanocrystal system at $T / T_{\text {gel }}=0.89$, with the same color coding as in (A). (C) Effective links per nanocrystal for temperature $T / T_{\text {gel }}=1.16,1.10,1.05$, and 0.89 (black circles, blue squares, purple diamonds, and orange triangles, respectively). (D) Maximum nanocrystal cluster size, with large clusters forming above the threshold of 1.0 effective link per nanocrystal.

The standard reaction enthalpy derived from van 't Hoff analysis (ln $K_{e q}$ vs. 1/T), independent of [TBACl], is $-45.8 \pm 0.3 \mathrm{~kJ} \mathrm{~mol}^{-1}$ (Fig. 4C). As expected, the standard reaction entropy is also approximately independent of [TBACl], within the precision of our analysis (fig. S29 and table S1). The derived enthalpy value is similar to the enthalpy calculated for DNA links 
made with six complementary base pairs (43). Here, this same bond strength is found in a relatively compact metal coordination complex, compatible with organic solvents, and stable during repeated thermal cycling. Furthermore, considering a $T_{g e l}$ of $50{ }^{\circ} \mathrm{C}$, the free energy for link formation is $-60.7 \mathrm{~kJ} \mathrm{~mol}^{-1}$, very close to the bonding energy predicted by the simulations $\left(57.8 \mathrm{~kJ} \mathrm{~mol}^{-1}\right)$. The $\mathrm{Co}(\mathrm{Tpy})_{2}$ linkages are also chemically responsive. As in previous studies of terpyridine links, the metal ions can be extracted by adding EDTA, recovering a nanocrystal dispersion with no evidence of remaining aggregation (fig. S30).

\section{DISCUSSION}

These thermoreversible gels offer a glimpse of the broadly tunable, responsive materials that can be envisioned, based on the microscopic bonding conditions established by our spectroscopic and computational analysis. Metal-terpyridine links are one of a family of dynamic covalent bonding chemistries that bond selectively only with their complementary pair, named as tunable orthogonal reversible covalent (TORC) bonds (44). We expect TORC linkages to be useful for selective "programming" of nanocrystal assembly, for example, TL-ITO could be selectively assembled from a mixed dispersion also containing nanocrystals functionalized with the aldehyde-functional ligands reported previously (8). The organization of multiple types (sizes, compositions, shapes) of nanocrystals within a gel network could be controlled dynamically, based on the linkers employed. Linking chemistries responsive to alternative stimuli, such as light, magnetic field, or $\mathrm{pH}$ changes, could be used. Besides optical properties, such assemblies could have switchable rheological characteristics or could be used to reverisibly trap and release drugs or other cargo (45). Finally, the microscopic insights into linking-controlled assembly afforded by our colorimetric chemistry may translate to other domains, e.g., understanding how thermodynamics controls protein assembly induced by metal ion linkers (46). 


\section{MATERIALS AND METHODS}

\section{Synthesis of ligand}

Terpyridine-terminated ligands (TL) were prepared by altering previously established literature protocols (8). Briefly, the ligands were made using solid phase peptide synthesis (SPPS), and the terminal terpyridine moiety was incorporated using copper azide alkyne cycloaddition (CuAAC). The ligand was built off of solid support and azido lysine was used during the SPPS to incorporate a terminal azide functional group. Then, terpyridine-based alkyne moiety was attached to the ligand $\mathrm{CuAAC}$. Once completed, the ligands were cleaved from the resin, purified using high performance liquid chromatography, and dried for use.

\section{Synthesis and functionalization of nanocrystals}

ITO nanocrystals were synthesized on a Schlenk line via a simple modification to a slow growth procedure (34). A precursor solution was prepared by dissolving $\operatorname{Sn}(\mathrm{IV})$ acetate $(117.4 \mathrm{mg}, 0.5$ $\mathrm{mmol})$ and $\mathrm{In}(\mathrm{III})$ acetate $(2.773 \mathrm{~g}, 9.5 \mathrm{mmol})$ in $20 \mathrm{~mL}$ oleic acid, degassing under vacuum (90 $\left.{ }^{\circ} \mathrm{C}, 1 \mathrm{~h}\right)$, and heating under flowing nitrogen $\left(150{ }^{\circ} \mathrm{C}, 3 \mathrm{~h}\right)$. The precursor solution was slowly injected into $13 \mathrm{~mL}$ oleyl alcohol at $290{ }^{\circ} \mathrm{C}$ under flowing nitrogen. Finally, the nanocrystals were washed five times (four times with ethanol and once with isopropanol) and dispersed in hexane.

The nanocrystals were functionalized via a direct ligand exchange (8). As-synthesized nanocrystals were precipitated into a pellet and sonicated in ligand solution $(0.01 \mathbf{M} \mathbf{T L}$ in DMF) until a clear dispersion was obtained. The functionalized nanocrystals were washed three times and dispersed in DMF.

\section{Preparation of samples for spectroscopic measurement}

The samples were prepared by adding $\mathrm{Co}^{2+}\left(0.01 \mathrm{M}\right.$ anhyd. $\mathrm{CoCl}_{2}$ in $\left.\mathrm{DMF}\right)$ and $\mathrm{Cl}^{-}(0.5$ $\mathrm{M}$ TBACl in DMF) to a stock solution of TL-ITO (43.0 mg/mL, in DMF). The final volume fraction of nanocrystal was adjusted to 0.001 by adding neat DMF. The final concentration of 
$\mathrm{CoCl}_{2}$ was fixed at $0.5 \mathrm{mM}$, while that of $\mathrm{TBACl}$ was varied between samples $([\mathrm{TBACl}]=100$, 150, and $200 \mathrm{mM}$ ). Control samples were prepared using nanocrystals capped with a ligand lacking the terpyridine group, replacing it with a phenyl ring, in otherwise the same conditions.

\section{Deconvolution of in situ absorption spectra}

The visible absorption spectra of nanocrystal dispersions were deconvoluted into 3 elements: ITO nanocrystals (bandgap and LSPR), bound $\mathrm{Co}^{2+}$, and free $\mathrm{Co}^{2+}$. First, the contribution from ITO nanocrystals was removed by subtracting the absorption spectrum of a TL-ITO dispersion, leaving the total absorption of bound and free $\mathrm{Co}^{2+}$. Next, the peak intensity of deconvoluted free $\mathrm{Co}^{2+}$ at $693 \mathrm{~nm}\left(I_{s}\right)$ was determined by using an extrapolated linear fit as a baseline. Meanwhile, in situ visible absorption of control samples were measured and their intensity at $693 \mathrm{~nm}$ $\left(I_{o}\right)$ was found. The deconvoluted spectroscopic contribution of free $\mathrm{Co}^{2+}$ was obtained by multiplying the intensity of the absorption spectra from the control samples with the factor of $I_{s} / I_{o}$. Lastly, the deconvoluted spectroscopic contribution of bound $\mathrm{Co}^{2+}$ was obtained by subtracting the contribution of free $\mathrm{Co}^{2+}$ from the $\mathrm{Co}^{2+}$ portion of the spectra.

The fractions of bound $\mathrm{Co}^{2+}$ at different temperatures were derived from the deconvoluted spectra. In the control samples, the high molar ratio of $\mathrm{Cl}^{-}$to $\mathrm{Co}^{2+}$ (ca. 200 to 400) favors free $\mathrm{Co}^{2+}$, yielding the $I_{o}$ values used for quantification. In the presence of TL-ITO, the amount of free $\mathrm{Co}^{2+}$ decreases as $\mathrm{Co}^{2+}$ bonds with terpyridine, resulting in reduced peak intensity $I_{s}$. Thus, the absorption intensity ratio, $I_{s} / I_{o}$, can be interpreted as the fraction of free $\mathrm{Co}^{2+}$ relative to the number of total $\mathrm{Co}^{2+}$ ions. The bound fraction is taken as the remainder of the $\mathrm{Co}^{2+}$ ions, readily calculated as $\left(I_{o}-I_{S}\right) / I_{o}$.

\section{MD simulation}

The coarse-grained model studied here via MD simulations was designed (8) to capture theoretically predicted bonding motifs (30-32) and experimentally observed phase behavior $(8,12)$ of linker-mediated colloidal nanocrystal dispersions and gels. Nanocrystals, ligands, and linkers 
are represented explicitly in the model with physical dimensions and bonding attributes approximating their counterparts in the experiments, while the solvent was treated implicitly (fig. S27). Molecular dynamics simulations were performed with LAMMPS (9 January 2020) (47), and Ovito (48) was used to visualize our simulations and to render the images and videos.

Details of the model and the simulations are provided in the Supplementary Information. A more extensive discussion of the model, including its approximations and a motivation for its paramterization can be found elsewhere (8). 


\section{REFERENCES AND NOTES}

1. D. V. Talapin, J.-S. Lee, M. V. Kovalenko, E. V. Shevchenko, Prospects of colloidal nanocrystals for electronic and optoelectronic applications. Chem. Rev. 110, 389-458 (2010).

2. A. Agrawal, S. H. Cho, O. Zandi, S. Ghosh, R. W. Johns, D. J. Milliron, Localized surface plasmon resonance in semiconductor nanocrystals. Chem. Rev. 118, 3121-3207 (2018).

3. J. Shamsi, A. S. Urban, M. Imran, L. De Trizio, L. Manna, Metal halide perovskite nanocrystals: Synthesis, post-synthesis modifications, and their optical properties. Chem. Rev. 119, 3296-3348 (2019).

4. A. Taleb, C. Petit, M. Pileni, Optical properties of self-assembled 2D and 3D superlattices of silver nanoparticles. J. Phys. Chem. B 102, 2214-2220 (1998).

5. C.-F. Chen, S.-D. Tzeng, H.-Y. Chen, K.-J. Lin, S. Gwo, Tunable plasmonic response from alkanethiolate-stabilized gold nanoparticle superlattices: Evidence of near-field coupling. J. Am. Chem. Soc. 130, 824-826 (2008).

6. Z. Nie, A. Petukhova, E. Kumacheva, Properties and emerging applications of selfassembled structures made from inorganic nanoparticles. Nat. Nanotechnol. 5, 15-25 (2010).

7. C. A. Saez Cabezas, G. K. Ong, R. B. Jadrich, B. A. Lindquist, A. Agrawal, T. M. Truskett, D. J. Milliron, Gelation of plasmonic metal oxide nanocrystals by polymer-induced depletion attractions. Proc. Natl. Acad. Sci. 115, 8925-8930 (2018).

8. M. N. Dominguez, M. P. Howard, J. M. Maier, S. A. Valenzuela, Z. M. Sherman, J. F. Reuther, L. C. Reimnitz, J. Kang, S. H. Cho, S. L. Gibbs, A. K. Menta, D. L. Zhuang, A. van der Stok, S. J. Kline, E. V. Anslyn, T. M. Truskett, D. J. Milliron, Assembly of linked nanocrystal colloids by reversible covalent bonds. Chem. Mater. 32, 10235-10245 (2020). 
9. A. Agrawal, A. Singh, S. Yazdi, A. Singh, G. K. Ong, K. Bustillo, R. W. Johns, E. Ringe, D. J. Milliron, Resonant coupling between molecular vibrations and localized surface plasmon resonance of faceted metal oxide nanocrystals. Nano Lett. 17, 2611-2620 (2017).

10. N. Gaponik, A.-K. Herrmann, A. Eychmüller, Colloidal nanocrystal-based gels and aerogels: Material aspects and application perspectives. J. Phys. Chem. Lett. 3, 8-17 (2012).

11. P. Rusch, D. Zámbó, N. C. Bigall, Control over structure and properties in nanocrystal aerogels at the nano-, micro-, and macroscale. Acc. Chem. Res. 53, 2414-2424 (2020).

12. Z. M. Sherman, A. M. Green, M. P. Howard, E. V. Anslyn, T. M. Truskett, D. J. Milliron, Colloidal nanocrystal gels from thermodynamic principles. Acc. Chem. Res. 54, 798-807 (2021).

13. T. Gacoin, K. Lahlil, P. Larregaray, J.-P. Boilot, Transformation of CdS colloids: Sols, gels, and precipitates. J. Phys. Chem. B 105, 10228-10235 (2001).

14. J. L. Mohanan, I. U. Arachchige, S. L. Brock, Porous semiconductor chalcogenide aerogels. Science 307, 397-400 (2005).

15. I. U. Arachchige, S. L. Brock, Highly luminescent quantum-dot monoliths. J. Am. Chem. Soc. 129, 1840-1841 (2007).

16. D. Wen, W. Liu, D. Haubold, C. Zhu, M. Oschatz, M. Holzschuh, A. Wolf, F. Simon, S. Kaskel, A. Eychmüller, Gold aerogels: Three-dimensional assembly of nanoparticles and their use as electrocatalytic interfaces. ACS Nano 10, 2559-2567 (2016).

17. I. U. Arachchige, S. L. Brock, Sol-gel methods for the assembly of metal chalcogenide quantum dots. Acc. Chem. Res. 40, 801-809 (2007).

18. Y. Wang, P. J. Santos, J. M. Kubiak, X. Guo, M. S. Lee, R. J. Macfarlane, Multistimuli responsive nanocomposite tectons for pathway dependent self-assembly and acceleration of covalent bond formation. J. Am. Chem. Soc. 141, 13234-13243 (2019). 
19. N. Marro, F. Della Sala, E. R. Kay, Programmable dynamic covalent nanoparticle building blocks with complementary reactivity. Chem. Sci. 11, 372-383 (2020).

20. B. A. Lindquist, R. B. Jadrich, D. J. Milliron, T. M. Truskett, On the formation of equilibrium gels via a macroscopic bond limitation. J. Chem. Phys. 145, 074906 (2016).

21. A. Singh, B. A. Lindquist, G. K. Ong, R. B. Jadrich, A. Singh, H. Ha, C. J. Ellison, T. M. Truskett, D. J. Milliron, Linking semiconductor nanocrystals into gel networks through all-inorganic bridges. Angew. Chem. Int. Ed. 54, 14840-14844 (2015).

22. V. Sayevich, B. Cai, A. Benad, D. Haubold, L. Sonntag, N. Gaponik, V. Lesnyak, A. Eychmüller, 3D assembly of all-inorganic colloidal nanocrystals into gels and aerogels. Angew. Chem. Int. Ed. 55, 6334-6338 (2016).

23. H. Xiong, D. van der Lelie, O. Gang, DNA linker-mediated crystallization of nanocolloids. J. Am. Chem. Soc. 130, 2442-2443 (2008).

24. J. J. Storhoff, A. A. Lazarides, R. C. Mucic, C. A. Mirkin, R. L. Letsinger, G. C. Schatz, What controls the optical properties of DNA-linked gold nanoparticle assemblies? J. Am. Chem. Soc. 122, 4640-4650 (2000).

25. S. Borsley, E. R. Kay, Dynamic covalent assembly and disassembly of nanoparticle aggregates. Chem. Commun. 52, 9117-9120 (2016).

26. B. L. Norsten, T. B. Frankamp, V. M. Rotello, Metal directed assembly of terpyridinefunctionalized gold nanoparticles. Nano Lett. 2, 1345-1348 (2002).

27. V. Lesnyak, S. V. Voitekhovich, P. N. Gaponik, N. Gaponik, A. Eychmüller, CdTe nanocrystals capped with a tetrazolyl analogue of thioglycolic acid: Aqueous synthesis, characterization, and metal-assisted assembly. ACS Nano 4, 4090-4096 (2010). 
28. A. Hitihami-Mudiyanselage, K. Senevirathne, S. L. Brock, Bottom-up assembly of $\mathrm{Ni}_{2} \mathrm{P}$ nanoparticles into three-dimensional architectures: An alternative mechanism for phosphide gelation. Chem. Mater. 26, 6251-6256 (2014).

29. Y. Sun, N. C. Harris, C.-H. Kiang, The reversible phase transition of DNA-linked colloidal gold assemblies. Physica A 354, 1-9 (2005).

30. M. P. Howard, R. B. Jadrich, B. A. Lindquist, F. Khabaz, R. T. Bonnecaze, D. J. Milliron, T. M. Truskett, Structure and phase behavior of polymer-linked colloidal gels. J. Chem. Phys. 151, 124901 (2019).

31. M. P. Howard, Z. M. Sherman, D. J. Milliron, T. M. Truskett, Wertheim's thermodynamic perturbation theory with double-bond association and its application to colloid-linker mixtures. J. Chem. Phys. 154, 024905 (2021).

32. M. P. Howard, Z. M. Sherman, A. N. Sreenivasan, S. A. Valenzuela, E. V. Anslyn, D. J. Milliron, T. M. Truskett, Effects of linker flexibility on phase behavior and structure of linked colloidal gels. J. Chem. Phys. 154, 074901 (2021).

33. S. H. Cho, K. M. Roccapriore, C. K. Dass, S. Ghosh, J. Choi, J. Noh, L. C. Reimnitz, S. Heo, K. Kim, K. Xie, B. A. Korgel, X. Li, J. R. Hendrickson, J. A. Hachtel, D. J. Milliron, Spectrally tunable infrared plasmonic F,Sn: $\operatorname{In}_{2} \mathrm{O}_{3}$ nanocrystal cubes. J. Chem. Phys. 152, 014709 (2020).

34. A. W. Jansons, J. E. Hutchison, Continuous growth of metal oxide nanocrystals: Enhanced control of nanocrystal size and radial dopant distribution. ACS Nano 10, 6942-6951 (2016).

35. E. Carbonell, L. A. Bivona, L. Fusaro, C. Aprile, Silsesquioxane-terpyridine nano building blocks for the design of three-dimensional polymeric networks. Inorg. Chem. 56, 63936403 (2017). 
36. L. Wang, B. Song, S. Khalife, Y. Li, L.-J. Ming, S. Bai, Y. Xu, H. Yu, M. Wang, H. Wang, X. Li, Introducing seven transition metal ions into terpyridine-based supramolecules: Selfassembly and dynamic ligand exchange study. J. Am. Chem. Soc. 142, 1811-1821 (2020).

37. J. De Roo, N. Yazdani, E. Drijvers, A. Lauria, J. Maes, J. S. Owen, I. Van Driessche, M. Niederberger, V. Wood, J. C. Martins, I. Infante, Z. Hens, Probing solvent-ligand interactions in colloidal nanocrystals by the NMR line broadening. Chem. Mater. 30, 5485-5492 (2018).

38. A. Winter, M. D. Hager, G. R. Newkome, U. S. Schubert, The marriage of terpyridines and inorganic nanoparticles: Synthetic aspects, characterization techniques, and potential applications. Adv. Mater. 23, 5728-5748 (2011).

39. Y. Yamanoi, Y. Yamamoto, M. Miyachi, M. Shimada, A. Minoda, S. Oshima, Y. Kobori, H. Nishihara, Nanoparticle assemblies via coordination with a tetrakis(terpyridine) linker bearing a rigid tetrahedral core. Langmuir 29, 8768-8772 (2013).

40. K. Schöller, C. Toncelli, J. Experton, S. Widmer, D. Rentsch, A. Vetushka, C. J. Martin, M. Heuberger, C. E. Housecroft, E. C. Constable, L. F. Boesel, L. J. Scherer, 2,2':6',2”terpyridine-functionalized redoxresponsive hydrogels as a platform for multiresponsive amphiphilic polymer membranes. RSC Adv. 6, 97921-97930 (2016).

41. C. C. Collins, S. Regalado-Love, R. I. Portillo, D. J. Boston, M. P. Shores, I. Bhowmick, Functionalized cellulose-co(ii)-bis-terpyridine hybrid material as colorimetric sensor for micromolar aqueous cyanide. Adv. Mater. Technol. 4, 1800406 (2019).

42. S. K. Ghosh, T. Pal, Interparticle coupling effect on the surface plasmon resonance of gold nanoparticles: From theory to applications. Chem. Rev. 107, 4797-4862 (2007).

43. J. SantaLucia, D. Hicks, The thermodynamics of DNA structural motifs. Annu. Rev. Biophys. Biomol. Struct. 33, 415-440 (2004). 
44. J. F. Reuther, S. D. Dahlhauser, E. V. Anslyn, Tunable orthogonal reversible covalent (TORC) bonds: Dynamic chemical control over molecular assembly. Angew. Chem. Int. Ed. 53, 74-85 (2018).

45. E. A. Appel, M. W. Tibbitt, M. J. Webber, B. A. Mattix, O. Veiseh, R. Langer, Selfassembled hydrogels utilizing polymer-nanoparticle interactions. Nat. Commun. 6, 6295 (2015).

46. E. N. Salgado, R. J. Radford, F. A. Tezcan, Metal-directed protein self-assembly. Acc. Chem. Res. 43, 661-672 (2010). PMID: 20192262.

47. S. Plimpton, Fast parallel algorithms for short-range molecular dynamics. J. Comp. Phys. 117, 1-19 (1995).

48. A. Stukowski, Visualization and analysis of atomistic simulation data with OVITO-the open visualization tool. Model. Simul. Mater. Sci. Eng. 18, 015012 (2009).

Acknowledgment: We acknowledge early conceptual contributions by Dr. Evan Runnerstrom. We would like to thank the University of Texas at Austin Mass Spectrometry and NMR Facility for the use of the Bruker AVANCE III 500: NIH grant number 1 S10 OD021508-01 and the Texas Materials Institute, for the use of the SAXSLAB Ganesha, acquired using an NSF MRI grant CBET-1624659. We thank the Texas Advanced Computing Center (TACC) at the University of Texas at Austin for HPC resources. Funding: This research was primarily supported by the National Science Foundation through the Center for Dynamics and Control of Materials: an NSF Materials Research Science and Engineering Center (NSF MRSEC) under Cooperative Agreement DMR-1720595. E. V. A. acknowledges support from the Welch Regents Chair (F-0046). D. J. M. and T. M. T. also acknowledge support by the Welch Foundation (F-1696 and F-1848). This work was also supported by an NSF Graduate Research Fellowships (DGE1610403) to S. A. V. and Arnold O. Beckman Postdoctoral Fellowship to Z. M. S. Author 
contributions: J. K. and S. A. V. contributed equally to this work. J. K. and D. J. M. conceived the idea and designed the research. J. K., T. M. T., E. V. A., and D. J. M. guided the research. J. K. performed synthesis of nanocrystals, material characterization, and in situ spectroscopy. S. A. V. designed, synthesized, and characterized ligands. E. Y. L. performed MD simulations. M. N. D. conducted ${ }^{19}$ F NMR spectroscopy measurement. J. K., D. J. M., S. A. V., T. M. T., and E. Y. L. wrote the manuscript with input from all authors. Competing interests: The authors declare no competing interests. Data and materials availability: All data needed to evaluate the conclusions in the paper are present in the paper and/or the Supplementary Materials. 
GRAPHICAL ABSTRACT

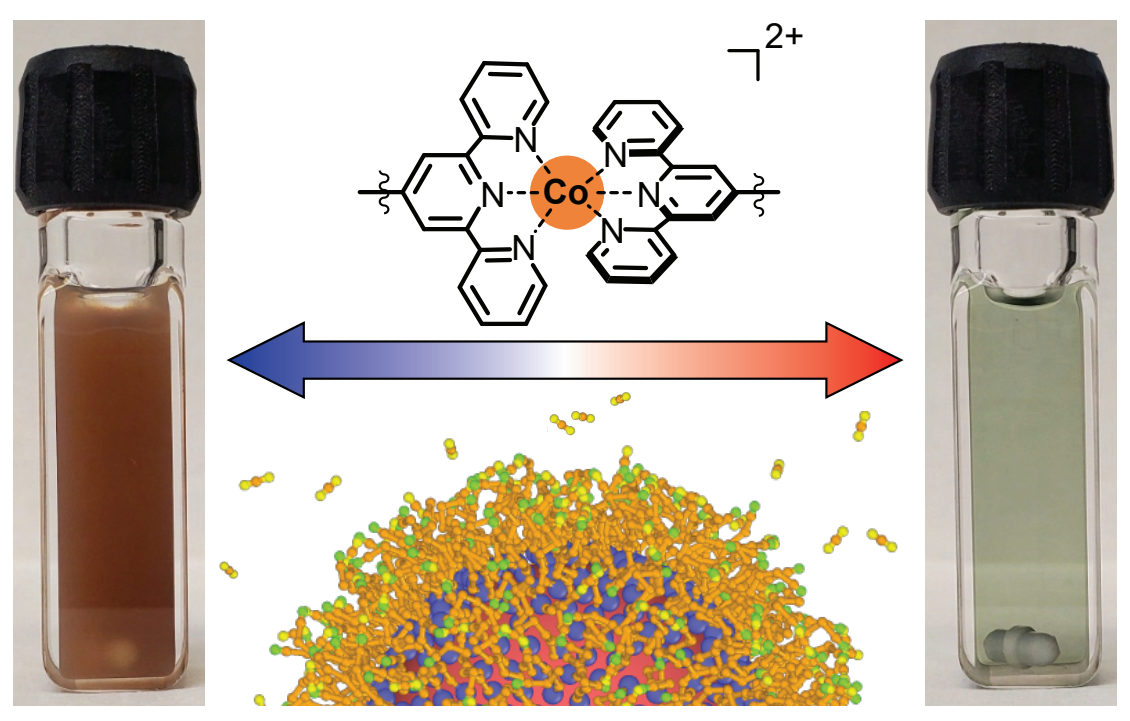

